Cahiers québécois de démographie

Revue internationale d'étude des populations

\title{
Les défis d'une nomenclature commune des professions pour l'étude de la mobilité intergénérationnelle en France et au Québec
}

\section{Challenges of a common nomenclature for coding professions to enable comparative study of intergenerational mobility in France and Quebec}

\author{
Delphine Remillon, Marianne Kempeneers et Eva Lelièvre
}

Volume 47, numéro 1, printemps 2018

L’apport du longitudinal dans l'analyse des inégalités

URI : https://id.erudit.org/iderudit/1062105ar

DOI : https://doi.org/10.7202/1062105ar

Aller au sommaire du numéro

Éditeur(s)

Association des démographes du Québec

ISSN

1705-1495 (numérique)

Découvrir la revue

Citer cet article

Remillon, D., Kempeneers, M. \& Lelièvre, E. (2018). Les défis d'une nomenclature commune des professions pour l'étude de la mobilité intergénérationnelle en France et au Québec. Cahiers québécois de démographie, 47(1), 25-48. https://doi.org/10.7202/1062105ar
Résumé de l'article

Il existe en France une longue tradition d'étude de la mobilité sociale, mais pendant longtemps les processus de transmission professionnelle au sein des familles n'ont été étudiés que de père en fils. Au Canada, ces analyses sont moins fréquentes, en raison du peu de données disponibles et parce que les nomenclatures se prêtent difficilement à un codage hiérarchique des professions. Tirant parti des enquêtes Biographies et entourage (INED) et Biographies et solidarités familiales au Québec (Université de Montréal), nous pouvons étudier les transmissions sur plusieurs générations de façon comparative entre la France et le Québec et identique pour les femmes et les hommes. Il a d'abord fallu coder les professions d'une manière comparable et adaptée à l'étude de la mobilité sociale. L'appui sur la nomenclature française, qui décrit les structures socioprofessionnelles de façon stable dans le temps, a permis de recréer des catégories hiérarchiques pour le Québec également. Le présent article rend compte de ces questions méthodologiques et du potentiel de ces deux enquêtes pour analyser la mobilité sociale. 
Cahiers québécois de démographie Vol. 47, ${ }^{\circ}$ 1, printemps 2018, p. 25-48

\title{
Les défis d'une nomenclature commune des professions pour l'étude de la mobilité intergénérationnelle en France et au Québec'
}

\author{
DELPHINE REMILLON*, MARIANNE KEMPENEERS ${ }^{\star *}$ ET EVA LELIÈVRE**
}

\begin{abstract}
RÉSUMÉ
Il existe en France une longue tradition d'étude de la mobilité sociale, mais pendant longtemps les processus de transmission professionnelle au sein des familles n'ont été étudiés que de père en fils. Au Canada, ces analyses sont moins fréquentes, en raison du peu de données disponibles et parce que les nomenclatures se prêtent difficilement à un codage hiérarchique des professions. Tirant parti des enquêtes Biographies et entourage (INED) et Biographies et solidarités familiales au Québec (Université de Montréal), nous pouvons étudier les transmissions sur plusieurs générations de façon comparative entre la France et le Québec et identique pour les femmes et les hommes. Il a d'abord fallu coder les professions d'une manière comparable et adaptée à l'étude de la mobilité sociale. L'appui sur la nomenclature française, qui décrit les structures socioprofessionnelles de façon stable dans le temps, a permis de recréer des catégories hiérarchiques pour le Québec également. Le présent article rend compte de ces questions méthodologiques et du potentiel de ces deux enquêtes pour analyser la mobilité sociale.
\end{abstract}

\begin{abstract}
Challenges of a common nomenclature for coding professions to enable comparative study of intergenerational mobility in France and Quebec

There is a long tradition in France of studying social mobility, but for a long time the processes of professional transmission within families were studied only from father to son. In Canada, social mobility analyses are much less frequent due to the limited data available and difficulties in coding occupations hierarchically. Taking advantage of INED's Biographies et entourage survey and the University of Montreal survey

1. Une première version de cet article a été présentée au congrès de l'ACFAS «L'apport du longitudinal dans l'analyse des inégalités », organisé par Solène Lardoux et Jean Poirier, et au séminaire des Lundis de l'INED. Nous remercions les organisateurs et les discutants, Stéphane Moulin et Milan Bouchet-Valat, ainsi que trois relecteurs anonymes et le comité de rédaction des Cahiers québécois de démographie pour l'ensemble de leurs remarques et suggestions. Un grand merci également à Isabelle Van de Pevenage pour son aide précieuse sur les premiers traitements et codages des professions dans l'enquête Biographies et solidarités familiales au Québec.

* Institut national d'études démographiques (INED), F-75020 Paris, France; LiRIS (Université de Rennes 2) ; Centre d'études de l'emploi et du travail (CEET); delphine.remillon@ined.fr.

** Département de sociologie, Université de Montréal, Québec, Canada; marianne.kempeneers@umontreal.ca.

*** Institut national d'études démographiques (INED), F-75020 Paris, France; eva@ined.fr.
\end{abstract}


Biographies and Family Solidarity in Quebec, we can study professional transmission across generations in a comparative way between France and Québec, taking into account both women and men. But first of all, there was a need to code professions in a comparable way allowing an analysis of social mobility. The use of the French nomenclature, which clearly depicts hierarchical socio-professional structures in a stable manner, has made it possible to recreate hierarchical categories for Quebec as well. This article reports on these methodological issues and the potential of these two surveys to address the issue of social mobility.

\section{INTRODUCTION}

$\mathrm{C}$ ontrairement à la France qui possède une tradition de longue date dans l'étude de la mobilité sociale, le Québec s'est relativement peu attaché à cette question. Une des raisons à cela est que les données statistiques dont dispose le Canada se prêtent difficilement aux études de mobilité sociale, bien que plusieurs chercheurs aient déploré cet état de fait au fil du temps et aient proposé des alternatives ponctuelles (voir Langlois, 2003).

Par le biais d'une enquête menée au Québec en 2004 (Biographies et solidarités familiales au Québec), nous disposons d'informations sur les professions de trois lignées, à savoir celles de générations nées au Québec entre 1934 et 1954, celles de leurs parents nés au début du siècle et celles de leurs enfants nés entre 1957 et 1988, de façon identique pour les femmes et pour les hommes (Kempeneers et Van Pevenage, 2011). Cette enquête s'inscrivait dans le sillage de l'enquête Biographies et Entourage réalisée par l'Ined en 2001, laquelle avait recueilli les mêmes données du côté français (Bonvalet et Lelièvre, 2012). C’est à partir de ces deux enquêtes qu'a été menée la mise en perspective des transmissions intergénérationnelles dans les deux pays dont il sera question ici. La démarche a nécessité un processus ardu de déconstruction/reconstruction des catégories en usage du côté québécois, car c'est seulement à ce prix que la comparaison devenait possible. Ce faisant, est apparu le potentiel heuristique de ces données d'enquête pour l'analyse de la mobilité sociale au Québec. Ainsi, l'objectif ici est surtout de faire état de ces enjeux et questions méthodologiques en prenant appui sur les premiers résultats de la comparaison.

À notre connaissance, il n'existe pas de travaux comparatifs entre la France et le Québec sur la question de la mobilité sociale. Or, la comparaison nous semble intéressante car les deux contextes présentent de fortes similitudes, mais l'Amérique du Nord a la réputation d'être une région plus ouverte et mobile que le vieux continent européen. Le contexte institutionnel québécois est-il plus ou moins favorable à la mobilité sociale que le contexte français? Des travaux sur la perception des inégalités (cités par Langlois, 2003) ont montré que le Québec serait peut-être plus proche du modèle « corporatiste » européen (France, Allemagne) que du modèle «anglo-saxon » et qu'il y aurait de ce point de vue une différence entre le Québec et le reste du Canada, ce qui justifierait une comparaison entre le Québec et la France. Le Québec se rapproche-t-il davantage du modèle américain, ou du modèle français du point de vue de la reproduction sociale?

Les générations que nous étudions (qui couvrent en partie celle des baby-boomers) ont, des deux côtés de l'Atlantique, connu les mêmes grandes transformations : montée de l'activité salariée féminine ${ }^{2}$, tertiarisation de l'économie, diminution de l'emploi agricole, etc. Au Québec

2. Le taux d'activité féminine est passé, au Québec, de 26,5\% en 1961 à $60 \%$ en 2004; en France, de 28,2 \% à $65 \%$ sur la même période. 
comme en France, la féminisation du salariat a été concomitante d'un accroissement du niveau d'éducation des femmes. Dans les deux cas, il demeure aussi des inégalités de carrières entre femmes et hommes (inégalités d'emploi - les femmes sont davantage touchées par le temps partiel subi et les emplois précaires, inégalités salariales, ségrégation professionnelle, inégalités dans la prise en charge du travail domestique, etc.). De fortes différences existent aussi entre les deux contextes nationaux : les contrats de travail sont moins protecteurs au Québec qu'en France, les retours en formation en cours de carrière sont beaucoup plus fréquents (Kempeneers et collab. 2015; Moulin et collab. 2013), etc. Il est donc intéressant de comparer les carrières de ces générations entre les deux pays et ce qu'il en est des transmissions professionnelles et mobilités sociales intergénérationnelles au sein des lignées en prenant en compte de façon identique les trajectoires des femmes et des hommes, les origines paternelles et maternelles.

Nous commencerons par présenter les nomenclatures des catégories professionnelles traditionnellement utilisées en France et au Québec et dans lesquelles les données des deux enquêtes avaient été initialement codées : celle de l'Insee en France et celle de Statistique Canada au Québec. Après avoir décrit les deux enquêtes, nous détaillerons les recodages effectués pour permettre la comparaison. Puis, nous présenterons des premiers résultats sur l'évolution de la structure des emplois des femmes et des hommes au fil des générations en France et au Québec et enfin sur les transmissions professionnelles dans les lignées. Ceux-ci montrent de grandes proximités entre la France et le Québec concernant les mécanismes de reproduction sociale, dès lors que la même nomenclature est utilisée. L'usage de la nomenclature hiérarchique française sur les données québécoises conduit également à des résultats nouveaux qui diffèrent de ce que donne la nomenclature canadienne, notamment sur la ségrégation par sexe au sommet de la hiérarchie socioprofessionnelle.

\section{LA MESURE DE LA MOBILITÉ SOCIALE EN FRANCE ET AU QUÉbEC}

En France, il existe une tradition de longue date d'étude de la mobilité sociale reposant sur la nomenclature des catégories socioprofessionnelles, une nomenclature hiérarchisée et stable, créée en 1954 et qui n'a connu que deux changements : une refonte en 1982 et une rénovation mineure en 2003. Les travaux historiques montrent que son concepteur (Jean Porte) a adopté, à l'origine, une approche empirique plutôt que théorique (Amossé, 2013). La refonte de 1982 s'appuie sur une vaste consultation des partenaires sociaux qui aboutit à une correspondance accrue entre la nomenclature et les conventions collectives. Elle est aussi nourrie de travaux sociologiques et notamment de ceux de Pierre Bourdieu, avec comme conséquence que les notions de capital économique ou de capital culturel irriguent les nouvelles professions et catégories sociales (PCS). Celles-ci reposent toujours, toutefois, sur une approche que l'on peut qualifier de " réaliste » (Amossé, 2013) ou de " constructiviste » (Chauvel et collab. 2002). Les PCS s'appuient en effet sur les catégories indigènes considérées comme le reflet de «luttes de classement " (Amossé, 2013). C'est là une grande spécificité de la nomenclature socioprofessionnelle française. À l'inverse, d'autres pays ont suivi des approches beaucoup plus théoriques. C'est notamment le cas de la nomenclature britannique, qui, depuis la rénovation de 2001, est directement inspirée des travaux de John Goldthorpe, un auteur majeur dans le champ des études de la mobilité sociale (Filhon et 
collab.2013). De plus, alors que dans d'autres pays elle est cantonnée au milieu académique, en France, la nomenclature des PCS sert aussi bien aux chercheurs qu'aux acteurs économiques et sociaux ainsi qu'aux journalistes du fait des correspondances très fortes avec les découpages institutionnels de l'emploi, les conventions collectives et les appellations indigènes (Penissat, 2012). Le « jeu de cartes » imaginé par Luc Boltanski et Laurent Thévenot montrait bien la diffusion très large, dans tous les milieux sociaux et toutes les professions, de la nomenclature des PCS qui constitue véritablement en France un objet d'identification (Boltanski et Thévenot, 1983). Aussi, malgré de fréquentes critiques, remises en cause ${ }^{3}$ et quelques tentatives pour proposer d'autres catégorisations comme les échelles de prestige, la «catégorie socioprofessionnelle est irremplaçable » en France (Chauvel et collab., 2002: 159), notamment pour l'étude de la mobilité et de la reproduction sociale, et ne cesse de prouver sa pertinence empirique sur ces sujets (Amossé, 2012). En effet, si la nomenclature française n'est pas exempte de biais - elle reflète par exemple mieux et plus finement les mobilités et professions masculines que féminines (Amossé, 2004) — elle permet globalement une bonne description des hiérarchies socioprofessionnelles.

Au Québec, la mobilité intergénérationnelle a été beaucoup moins étudiée qu'en France, ou alors sous l'angle de la mobilité intergénérationnelle de revenus (par exemple Corak et Heisz, 1999). Deux facteurs ont sans doute contribué au faible développement de ce champ d'étude. D'une part, le statut historique particulier du Québec comme nation francophone dans l'ensemble canadien anglophone et d'autre part, la nature des données disponibles. Concernant le premier point, d'entrée de jeu se posait pour le Québec la difficile question de la relation entre classe et nation : comment saisir la dynamique de classes interne au Québec tout en prenant en compte le rapport inégalitaire entre anglophones (minoritaires sur le plan démographique mais dominants sur le plan économique) et francophones? De ce fait, quel univers circonscrire pour étudier les mécanismes de la mobilité sociale? Le Québec dans son ensemble incluant la minorité anglophone ou le Québec francophone seulement (Langlois, 2003) ? À ces considérations sociopolitiques s'ajoutait la question des données. Contrairement à la France, mais aussi à d'autres pays anglosaxons (Grande-Bretagne et États-Unis), la statistique canadienne dispose initialement d'une classification des professions que l'on peut qualifier de descriptive et technique plutôt que sociale et hiérarchisée en classes ou niveaux socioéconomiques. Il y a des raisons historiques à cela (Beaud et Prévost, 1992). Cette typologie combine profession et secteur d'activité et s'avère impossible à utiliser comme telle dans les études sociologiques, les regroupements étant trop indifférenciés et hétérogènes. Cette nomenclature a surtout servi à des études sur l'évolution à long terme de la structure de la main d'œuvre.

Pour étudier la mobilité sociale, il faut disposer d'instruments de mesure du statut social capables d'établir une hiérarchie entre les différentes professions. C'est à partir de la notion de "prestige» que diverses échelles ont été élaborées au fil du temps (Laroche, 1997). Dès 1950, le Code Rocher distinguait huit catégories de professions en fonction du prestige dont elles jouis-

3. Les critiques (Pierru et Spire, 2008) évoquent une nomenclature datée — « un outil de représentation du monde censé demeurer inchangé quand rien ne l'est » (Amossé, 2013 : 1040) ou encore l'imperfection (du point de vue de certains économètres à la recherche d'une "bonne variable ») de cette mesure multidimensionnelle (Brousse, 2012). 
saient dans la société québécoise (de profession libérale à agriculteur/ouvrier non qualifié). Cette échelle a été adaptée et utilisée par la suite par d'autres chercheurs (Langlois, 2003). En 1965, la classification de Pineo-Porter McRoberts (PPM) classait 204 professions selon le prestige (social standing) en 9 catégories socioéconomiques. En 1977, cette classification se complexifie et permet de classer les 486 « professions de base » de la Classification canadienne descriptive des professions (CCDP), introduite par Statistique Canada lors du recensement de 1971. Ces professions de base sont alors réparties en 16 catégories professionnelles. Une mise à jour de l'échelle PPM est effectuée en 1985 pour tenir compte des changements apportés en 1980 par Statistique Canada à la nomenclature des professions de la CCDP pour constituer la Classification type des professions. Cette version révisée de l'échelle PPM comporte également 16 catégories, mais une version condensée et révisée des catégories socioéconomiques est utilisée par Statistique Canada dans le cadre de l'Enquête sociale générale (ESG) de 1986. Viendra ensuite l'indice Blishen, Carroll, Moore (BCM), dérivé en partie de l'indice PPM mais qui prend également en compte le degré de scolarité et le niveau de revenu des personnes exerçant une profession. Cette échelle compte 514 professions et Statistique Canada modifie l'échelle de prestige PPM en fonction des scores de Blishen pour analyser les données sur la mobilité sociale de l'ESG de 1986 (Laroche, 1997 : 170). Depuis 2001, c'est principalement la nomenclature CNP (Classification nationale des professions) de Statistique Canada qui est utilisée. Elle divise l'ensemble des professions selon le genre de compétences et niveau des compétences requis pour accéder à un emploi et en remplir les fonctions, en prenant surtout en compte le domaine de formation et le niveau d'études et de responsabilités. Des correspondances peuvent être établies avec la classification PPM, mais « dans le détail, de nombreux changements sont opérés entre ces deux nomenclatures [...] ces changements témoignent à la fois de la difficulté de juger des niveaux de compétence professionnelle ainsi que des difficultés inhérentes à l'exercice de comparabilité des données historiques» (Moulin, 2016:280).

Ces jalons brièvement résumés de la définition et de la mesure de la mobilité sociale au Québec et au Canada mettent en évidence les problèmes de continuité qui se posent aujourd'hui face à des nomenclatures qui ont changé si souvent, rendant impossibles des analyses tant sur le long terme que sur l'axe intergénérationnel. On constate également que la question de l'origine sociale apparaît très peu dans les enquêtes canadiennes alors que c'est une question quasi systématique dans toutes les enquêtes françaises, y compris celles ne portant pas sur les questions de mobilité ou de reproduction sociale.

\section{MÉTHODES}

\subsection{Présentation des deux enquêtes et des variables professionnelles sélectionnées}

Les deux enquêtes sur lesquelles nous allons étayer ce travail ont été conduites dans l'optique commune d'explorer la dynamique des solidarités partagées et des liens au sein de la famille, qu'ils soient d'ordre résidentiel (proximité géographique, fréquence des contacts) ou professionnel. L'enquête Biographies et entourage de 2001 a été conduite auprès de 2830 individus. Elle est représentative des personnes habitant l'île-de-France en 1999 et nées entre 1930 et 1950. L'échantillonnage a sélectionné aléatoirement les communes enquêtées au sein de l'Île-de- 
France en tenant compte de leur composition socioprofessionnelle 4 , afin de refléter la structure socioprofessionnelle de la région capitale. Une pondération classique basée sur un calage par sexe, âge, statut matrimonial et lieu de résidence a ensuite pallié la non-réponse. L'enquête retrace les trajectoires familiale, résidentielle et professionnelle de la personne enquêtée et explore l'histoire de sa lignée (des grands-parents aux petits-enfants), de ses conjoints et de ses proches (Bonvalet et Lelièvre, 2012). L'enquête Biographies et solidarités familiales au Québec de 2004, inspirée de ces principes de collecte, a interrogé 502 personnes francophones, nées au Québec entre 1934 et 1954 et résidant dans la région du Grand Montréal5. L'échantillonnage a été constitué par un tirage aléatoire de numéros de téléphone et les enquêtes ont été réalisées en face à face. L'échantillon a été confronté aux données du recensement canadien de 2001. Quatre variables ont été utilisées pour le calcul des pondérations : sexe, âge, statut matrimonial et diplôme universitaire le plus élevé (Kempeneers et Van Pevenage, 2013). L'enquête reconstitue l'histoire familiale, professionnelle et résidentielle de la personne enquêtée, de ses conjoints et de ses proches. De plus, elle explore les moments-clés ayant mobilisé différentes formes de solidarité familiale (Kempeneers et Van Pevenage, 2011).

Les données collectées sur les carrières professionnelles et plus largement sur les trajectoires d'activité sont très détaillées pour les personnes enquêtées (relatées d'année en année de la fin des études à la date de l'enquête, voir Annexes) et sont sous une forme plus résumée concernant la carrière des parents, des conjoints et des enfants, car recueillies par proxy (voir Encadré 1 et Annexes). Ces données sont novatrices à plus d'un titre dans le champ des études sur la transmission intergénérationnelle des professions. Premièrement, concernant le Québec, elles se prêtent à l'établissement d'une nomenclature socioprofessionnelle sur un mode hiérarchique plutôt que technique. Deuxièmement, tant du côté québécois que du côté français, elles permettent non seulement de traiter trois générations (la plupart des études n'en abordent que deux) mais aussi d'articuler la profession de la personne enquêtée avec celle de son/sa ou ses conjoints ainsi que celles de ses frères et sœurs, ouvrant ainsi sur le concept de «trajectoires professionnelles familiales ». En revanche, ces enquêtes ne sont représentatives que des générations 1930-1950 résidant en Île-de-France d'un côté, et des générations 1934-1954 nées au Québec, francophones et résidant dans la région du Grand Montréal de l'autre et sont donc sujettes à des biais de sélection. Ces limites sont toutefois assez comparables d'une enquête à l'autre - mêmes générations enquêtées, habitants de deux grandes agglomérations — ce qui justifie la comparaison.

4. Définie selon une typologie des communes établie par l'Insee.

5. Au sens de la région métropolitaine de recensement, entité définie par Statistique Canada, correspondant à l'agglomération urbaine formée de la ville de Montréal et de sa grande banlieue. 
ENCADRÉ 1

Variables professionnelles retenues pour l'analyse des transmissions professionnelles France-Québec

Les éléments sur les carrières disponibles sont variables d'une enquête à l'autre. Ainsi, l'enquête québécoise comprend uniquement la profession principale pour tout autre membre de la lignée que la personne enquêtée (parents, frères et sœurs, conjoints et enfants). L'enquête française fournit plus de détail sur la carrière des parents et du ou des conjoints : plusieurs étapes sont décrites en relation avec les événements familiaux ayant jalonné leur vie (par exemple, pour les parents : première profession; profession à la naissance de la personne enquêtée (Ego); dernière profession, etc. Voir Annexes). S'ensuit une question formulée ainsi : «Récapitulons : pour vous, quelle a été son activité principale? » Pour les besoins de la comparaison, nous avons donc été amenées à sélectionner cette profession principale déclarée, pour faire le symétrique avec l'information disponible dans l'enquête québécoise ${ }^{6}$.

En revanche, des deux côtés, on ne dispose pas d'une variable synthétique de profession pour Ego, mais de son calendrier d'activité professionnelle détaillé, année par année. Il a donc fallu sélectionner une profession dans ce calendrier. Nous avons au départ retenu dans les deux cas la dernière profession ou position hors retraite ou inactivité temporaire du type maladie, préretraite, chômage, formation, etc. Cela conduisait à une proportion bien plus importante d'inactives chez les enquêtées françaises que chez les enquêtées québécoises $(24,5 \%$ contre $8,5 \%$ ). Or, si l'on regarde dans le détail leur trajectoire d'activité, les québécoises sont à peu de choses près aussi nombreuses que les Françaises à avoir connu au moins une période «au foyer » dans leur trajectoire ( $53 \%$ contre $59 \%$ ), mais elles ne finissent pas fréquemment leur trajectoire dans cette position contrairement aux Françaises. Le profil des trajectoires d'activité des femmes de ces générations diffère entre la France et le Québec (Kempeneers et collab. 2015). Pour ne pas surestimer le poids de l'inactivité dans les trajectoires des Françaises et récupérer un meilleur indicateur de leur milieu socioprofessionnel dans l'optique d'étudier les transmissions professionnelles, nous avons sélectionné leur dernière profession d'active. Les enquêtées dont la profession demeure « inactive » sont ainsi, du côté français comme du côté québécois, uniquement des femmes n'ayant jamais ou quasiment jamais eu d'activité professionnelle rémunérée (4,6 \% des Françaises et 7,3\% des Québécoises).

\subsection{Les codages effectués pour mesurer les transmissions professionnelles}

Dans l'enquête Biographies et solidarités familiales au Québec, la nomenclature dans laquelle les professions ont été codées initialement est la Classification nationale des professions pour statistiques (CNP-S) de 2001. Un code a été attribué à chaque profession, à 2 ou 4 chiffres en fonction de

6. Dans un article récent sur les seules données françaises, nous avons sélectionné une profession autre que la profession principale pour les parents et conjoints : la dernière profession d'actif ou d'active (Remillon et Lelièvre, 2018). La distribution des professions pour ces membres des lignées peut donc varier par rapport à ce que nous présentons ici. 
la précision d'information disponible: au moins toujours un intitulé de profession et la variable «à son compte ou salarié » et, pour Ego, quelques variables supplémentaires (nom et activité de l'entreprise, nombre d'employés et type de contrat temps plein/temps partiel, voir Annexes). Tout l'enjeu de la présente recherche a été, à partir de là, de reconstruire plusieurs variables pour pouvoir d'une part étudier l'évolution de la structure socioprofessionnelle au Québec au fil des générations (en ayant des données comparables aux travaux québécois existants afin de s'assurer, par une validation externe, de la qualité et de la cohérence des informations recueillies dans notre enquête) et d'autre part, pour pouvoir comparer les transmissions professionnelles entre la France et le Québec.

Le Schéma 1 résume les opérations de codage effectuées. Nous nous sommes d'abord servies des correspondances entre code CNP-S et code $\mathrm{CNP}^{7}$ pour reconstituer deux variables aujourd'hui traditionnellement utilisées pour décrire la structure de l'emploi au Canada : le genre de compétences et le niveau de compétences. Puis nous avons créé une variable de PCS sur le modèle français afin de permettre la comparaison.

SCHÉMA 1

Recodages effectués sur les professions québécoises

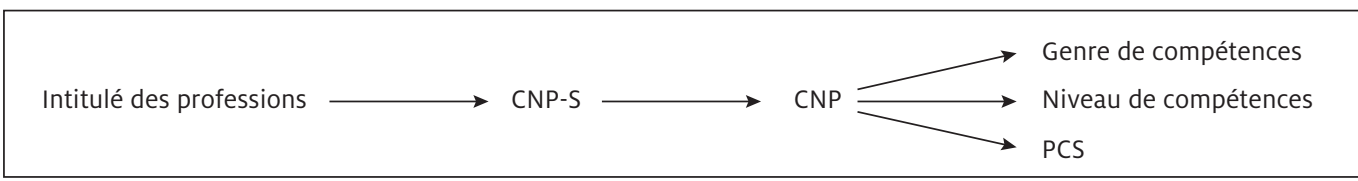

\subsubsection{Genre et niveau de compétences}

La variable genre de compétences (ou domaine d'activité), correspondant au premier chiffre du code $\mathrm{CNP}$, est fonction du domaine d'études donnant accès à cette profession et du secteur d'activités.

\begin{tabular}{|l|}
\hline o. Gestion \\
\hline 1. Affaires, finances, administration \\
\hline 2. Sciences naturelles et appliquées \\
\hline 3. Secteur de la santé \\
\hline 4. Sciences sociales, enseignement, administration publique et religion \\
\hline 5. Art, culture, sport et loisirs \\
\hline 6. Ventes et services \\
\hline 7. Métiers, transport et machinerie \\
\hline 9. Transformation, fabrication et services d'utilité publique \\
\hline
\end{tabular}

7. Jusqu'en 2011, la CNP et la CNP-S constituaient deux structurations des professions alternatives, utilisées, l'une par Ressources humaines et développement des compétences Canada et l'autre par Statistique Canada. Elles se différenciaient par la structure des grands groupes. Depuis 2011 les deux versions sont unifiées. 
La variable « niveau de compétences » correspondant au deuxième chiffre du code CNP, est défini selon le niveau des études et formations requises pour accéder à un emploi et en exercer les fonctions. Nous distinguons le niveau A, le niveau B et regroupons les niveaux C et D.

$\mathrm{A}=$ Professionnels et semi-professionnels

$\mathrm{B}=$ Techniciens, superviseurs, contremaîtres, travailleurs spécialisés

$\mathrm{C}-\mathrm{D}=$ Personnel de soutien et manœuvres, travailleurs semi et non spécialisés

Le niveau A regroupe les professions exigeant une formation universitaire (baccalauréat, maîtrise ou doctorat); le niveau $\mathrm{B}$, les professions qui exigent une formation collégiale (2 à 3 ans d'études postsecondaires dans un collège communautaire), un programme d'apprentissage ou un diplôme d'école des métiers. Ce niveau est aussi attribué à certaines professions exerçant des fonctions de supervision ou des responsabilités importantes dans le domaine de la santé et de la sécurité (pompiers, policiers, infirmières auxiliaires). Enfin, les niveaux $\mathrm{C}$ et $\mathrm{D}$ regroupent des professions de niveau de formation secondaire ou qui demandent une formation spécifique à la profession ou une formation en cours d'emploi (Moulin, 2016).

Pour effectuer ces deux codages, il a parfois été nécessaire de revenir à l'intitulé des professions car, jusqu'en 2011, il n'y a pas une stricte correspondance entre un code CNP-S à 2 chiffres et un code CNP (les grands groupes de la CNP-S représentent une agrégation statistique alternative de la CNP, voir ci-dessus). Un exemple de recodage "manuel » effectué est présenté dans l'Encadré 2.

La répartition des personnes enquêtées actives selon les deux variables de genre de compétences et de niveau de compétences est présentée dans les tableaux 1 et 2.

Les résultats sont globalement cohérents avec ce que l'on sait de la structure de l'emploi au Québec, avec seulement un peu plus d'individus dans les métiers des services et qualifiés que la moyenne, ce qui est normal du fait de la sélection de l'échantillon (Grand Montréal) ${ }^{8}$.

On retrouve, comme dans le reste du Québec, une forte ségrégation femmes/hommes par genre de compétences (Gauthier, 2001; Chevrier et Tremblay, 2003). Les hommes sont surreprésentés dans les domaines "Gestion », "Sciences naturelles et appliquées », " Métiers, transport et machinerie »; les femmes sont surreprésentées dans les domaines « Affaires, finances et administration ", "Secteur de la santé » et, dans une moindre mesure, dans le domaine "Sciences sociales, enseignement ». Ces écarts entre femmes et hommes sont statistiquement significatifs.

Comme en France (Amossé, 2004; Maruani, 2003; Marchand, 1993), on constate que davantage de femmes, par rapport aux hommes, sont concentrées dans certaines professions ou

8. Lorsque l'on compare au Portrait social du Québec de 2001 (Gauthier, 2001 : 247), on constate que l'on a, dans notre enquête, une proportion plus élevée que dans l'ensemble du Québec d'individus dans les catégories «Gestion », "Affaires, finances et administration », "Secteur de la santé », "Sciences sociales, enseignement, administration publique et religion » et «Arts, culture, sports et loisirs ». En revanche, les genres de compétences « Sciences naturelles et appliquées », "Ventes et services », " Métiers, transport et machinerie » et surtout, " Professions propres au secteur primaire » sont sous-représentés. La proportion de "professionnels» (niveau A de compétences) est également plus élevée. Outre la différence de date (1996 pour Le Portrait social contre 2004 pour notre enquête), la différence tient surtout au fait que notre enquête a porté sur une aire urbaine. Les écarts restent néanmoins relativement faibles, de l'ordre de 4 à 5 points maximum. 
TABLEAU 1

Répartition des Ego actifs et actives entre les genres de compétences (Québec) ${ }^{\mathrm{a} b}$

\begin{tabular}{|c|c|c|c|}
\hline \multirow[b]{2}{*}{ Genres de compétences } & \multicolumn{3}{|c|}{ \% pondérés } \\
\hline & Ensemble & Hommes & Femmes \\
\hline o. Gestion & 11,7 & 13,7 & 10,0 \\
\hline 1. Affaires, finances, administration & 24,9 & 17,8 & 31,1 \\
\hline 2. Sciences naturelles et appliquées & 4,2 & 7,1 & 1,6 \\
\hline 3. Secteur de la santé & 11,0 & 4,7 & 16,4 \\
\hline 4. Sciences sociales, enseignement, administration publique, religion & 12,5 & 10,7 & 14,1 \\
\hline 5. Art, culture, sport et loisirs & 6,5 & 7,4 & 5,8 \\
\hline 6. Ventes et services & 18,3 & 17,1 & 19,4 \\
\hline 7. Métiers, transport et machinerie & 10,4 & 20,6 & 1,6 \\
\hline 9. Transformation, fabrication et services d'utilité publique & 0,5 & 0,9 & 0,2 \\
\hline Ensemble des actifs et actives & 100,0 & 100,0 & 100,0 \\
\hline
\end{tabular}

a Actifs et actives francophones des générations 1934-1954, nés au Québec, résidant dans la région du Grand Montréal en 2004.

${ }^{b}$ L'interprétation de ce tableau se fait par exemple de la manière suivante: le dernier emploi de $13,7 \%$ des Ego hommes est dans le genre de compétences «Gestion » contre $10 \%$ de celui des femmes et $11,7 \%$ de celui des deux sexes. Les hommes sont donc surreprésentés dans ce domaine.

Source: Enquête Biographies et solidarités familiales au Québec (Université de Montréal, 2004)

TABLEAU 2

Répartition des Ego actifs et actives entre les niveaux de compétences (Québec) ${ }^{\mathrm{a} b}$

\begin{tabular}{|l|c|c|c|}
\hline & \multicolumn{3}{|c|}{$\%$ pondérés } \\
\hline Niveaux de compétences & Ensemble & Hommes & Femmes \\
\hline Niveau A - Professionnels et semi-professionnels & 27,2 & 25,8 & 28,4 \\
\hline Niveau B - Techniciens, superviseurs, contremaîtres, travailleurs spécialisés & 34,5 & 35,6 & 33,6 \\
\hline $\begin{array}{l}\text { Niveaux C-D - Personnel de soutien et manœuvres, travailleurs semi et non } \\
\text { spécialisés }\end{array}$ & 38,2 & 38,6 & 38,0 \\
\hline Ensemble des actifs et actives hors gestionnaires & 100,0 & 100,0 & 100,0 \\
\hline
\end{tabular}

a Actifs et actives francophones des générations 1934-1954, nés au Québec, résidant dans la région du Grand Montréal en 2004, hors gestionnaires car aucun niveau de compétences n'est distingué pour le genre de compétences o.

${ }^{b}$ L'interprétation de ce tableau se fait par exemple de la manière suivante: $25,8 \%$ des Ego hommes avait comme dernière profession une profession de niveau A contre $28,4 \%$ des femmes et $27,2 \%$ pour les deux sexes. Les femmes sont donc légèrement surreprésentées à ce niveau d'emploi.

Source: Enquête Biographies et solidarités familiales au Québec (Université de Montréal, 2004) 
domaines d'activité. Ainsi, les trois « genres de compétences» les plus fréquents pour chacun des sexes regroupent $55 \%$ des hommes contre $68 \%$ des femmes. Ces résultats sont confirmés par l'étude des 20 codes professionnels les plus fréquents dans l'échantillon : 25 enquêtées sont secrétaires et 21 sont infirmières. À l'inverse, le code de profession le plus fréquent chez les enquêtés («personnel administratif et de réglementation») ne regroupe que 6 hommes. Globalement, la structure de l'emploi est très différente entre les deux sexes et les hommes sont davantage représentés dans des emplois qualifiés. Pourtant, la nomenclature en " niveaux de compétences » reflète assez mal ces écarts. Certes, les femmes sont sous-représentées parmi les gestionnaires mais elles sont proportionnellement un peu plus nombreuses parmi les professionnels (niveau A) que les hommes. Globalement, les écarts femmes-hommes par niveaux de compétences sont faibles (et non statistiquement significatifs dans notre échantillon). Le résultat est-il le même si l'on utilise une nomenclature hiérarchique des emplois, la nomenclature française?

\subsubsection{Catégories socioprofessionnelles}

S'est en effet posée la question d'une nomenclature commune pour pouvoir comparer les deux enquêtes, française et québécoise. Dans l'enquête française Biographies et entourage, les professions étaient codées selon la nomenclature des PCS. Le niveau 1 des PCS françaises distingue six grandes catégories socioprofessionnelles selon le statut de l'emploi (indépendant/salarié) et le niveau de qualification : les deux premières catégories (agriculteurs/trices-exploitant.e.s et artisans-commerçant.e.s) sont des catégories d'indépendant.e.s. Étant donné les faibles effectifs concernant ces deux groupes dans nos échantillons, nous les regroupons dans une seule catégorie « indépendant.e.s».

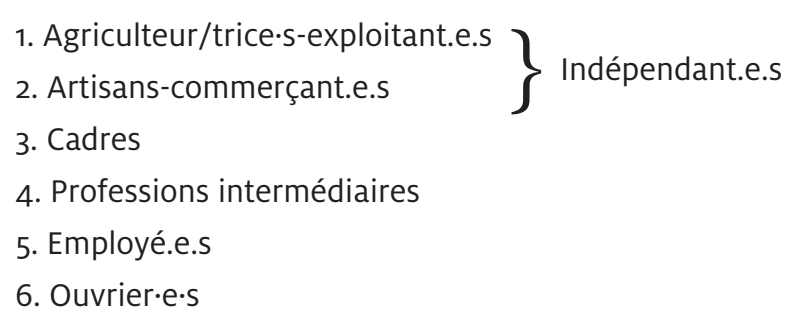

Nous avons choisi de construire une variable de PCS sur le modèle français pour l'enquête québécoise. L'avantage de répliquer la nomenclature française sur les données québécoises était de plusieurs ordres. Cela permettait entre autres d'utiliser une nomenclature hiérarchique et stable, donc adaptée aux études de mobilité sociale qui a fait ses preuves dans les travaux sur la reproduction sociale 9 . De plus, elle est, en ce qui a trait aux principes de construction, assez semblable à celles d'autres pays ${ }^{10}$. À l'inverse, la nomenclature canadienne des CNP est récente et est peu stable,

9. Cette nomenclature est considérée comme meilleure pour la comparaison intergénérationnelle que le revenu ou le diplôme car elle permet de coder de façon satisfaisante la situation socioprofessionnelle des ascendants des enquêtés, donc de personnes nées à une période décalée par rapport à celle de construction de la nomenclature (voir Chauvel et collab. 2002)

10. Notamment des pays européens avec lesquels ont été menées des recherches comparatives dans le cadre des travaux d'élaboration d'une nomenclature européenne ESec puis ESeG (voir Pénissat et collab. 2015; Filhon et collab. 2013; Penissat, 2012; Brousse, 2012, Méron et collab. 2016). 
elle n'est pas l'objet d'une identification forte des Québécois comme le sont les PCS françaises. Par ailleurs, cette nomenclature en niveaux de compétences souffre d'un manque d'homogénéité des catégories. Comme le montre Moulin (2016:305-309), il y a de fortes disparités salariales au sein de chacun des niveaux. En outre, le niveau retenu pour certaines professions ne correspond pas toujours de façon adéquate à la situation québécoise. Gauthier (2001 : 258) évoque la situation des infirmières, classées dans le niveau A alors qu'elles pourraient appartenir au niveau B car leur emploi n'exige pas un diplôme universitaire. En raison de la faiblesse des effectifs dans nos enquêtes, nous n'avons néanmoins pas pu effectuer un codage très fin des PCS (distinguer par exemple les ouvriers et employés qualifiés des non qualifiés). Les regroupements que nous proposons retiennent seulement le niveau le plus agrégé de la nomenclature française.

Souvent, la correspondance entre un code CNP-S et une PCS était immédiate. Par exemple, le code D1 de la CNP-S correspond aux infirmières, qui sont classées dans les professions intermédiaires dans la nomenclature des PCS. Il a néanmoins fallu revenir à l'intitulé des professions dans un certain nombre de cas, lorsque les professions regroupées dans un code CNP-S correspondaient à plusieurs PCS (voir Encadré 2).

\section{ENCADRÉ 2}

Exemple des recodages effectués

La grande catégorie G9 de la CNP-S de 2001, «Personnel de la vente et des services », contient des professions classées niveau B dans la CNP (coiffeurs, boulangers, bouchers, etc.) et d'autres classées aux niveaux C-D (serveurs, nettoyeurs, etc.). Il n'était donc pas possible, dans ce cas, de faire une correspondance directe entre ce code CNP-S et un code CNP. Il a alors fallu revenir à l'intitulé des professions pour déterminer le niveau de compétences de chaque individu appartenant à cette catégorie.

De même, si la plupart des professions que regroupe cette catégorie G9 de la CNP-S sont classées comme "employés » dans la nomenclature française des PCS (coiffeurs, concierges, serveurs, etc.), ce n'est pas le cas des bouchers et des boulangers. Ces professions étant largement masculines, elles ont été classées avec les ouvriers. Cela s'explique par le caractère relativement sexué des PCS françaises (Amossé, 2004). Là encore, il a fallu revenir au niveau de l'intitulé des professions pour faire les bons regroupements en PCS.

Avec les informations dont on disposait dans l'enquête québécoise, il n'était pas toujours possible de distinguer entre les enseignants de l'école primaire, classés comme "professions intermédiaires » dans les PCS, et les enseignants du secondaire et du supérieur, classés avec les « cadres ». Comme les premiers sont les plus nombreux, le choix a été fait de mettre tous les enseignants dans la catégorie " professions intermédiaires".

Le codage en PCS donne une autre image que le codage en niveaux de compétences de la répartition femmes-hommes dans la hiérarchie socioprofessionnelle. Alors que les femmes étaient surreprésentées dans les professions les plus qualifiées de la grille CNP (le niveau A), elles apparaissent, tout comme en France, sous-représentées dans le niveau «Cadres » qui est le niveau le 
plus élevé dans la nomenclature française (voir tableau 3) ${ }^{11}$. L'intérêt de mobiliser différentes catégorisations des professions comme nous le faisons ici est d'appréhender la pertinence des frontières de nos représentations (Chauvel et collab. 2002).

\section{3. ÉVOLUTION DE LA STRUCTURE SOCIOPROFESSIONNELLE DES EMPLOIS ET TRANSMISSIONS PROFESSIONNELLES AU SEIN DES LIGNÉES}

Une fois la nomenclature commune des professions construite, la comparaison des transmissions professionnelles devient possible. Préalablement, il convient de décrire la répartition dans les catégories socioprofessionnelles (les PCS) des femmes et des hommes des différentes générations (parents, Ego, enfants), en France et au Québec, afin d'avoir en tête l'évolution structurelle des professions qui détermine, en grande partie, la mobilité sociale. Nous présenterons ensuite quelques résultats sur les origines sociales des générations enquêtées, en France et au Québec.

\subsection{Répartition des positions socioprofessionnelles dans les lignées des générations enquêtées}

Les tableaux 3 et 4 présentent la répartition socioprofessionnelle des membres des différentes lignées, en séparant les femmes et les hommes, au Québec puis en France.

Du côté du Québec, on constate une forte baisse des indépendants de génération en génération chez les hommes, les femmes ayant toujours été très peu représentées dans cette position, même dans la génération des parents ${ }^{12}$. On voit aussi une hausse de la proportion de cadres, notamment entre la génération des parents et celle enquêtée, avec un rapprochement entre femmes et hommes au fil des générations, mais les hommes restent proportionnellement plus nombreux dans cette position, même parmi la génération des enfants aînés. Il faut toutefois garder en tête que les enfants encore en études au moment de l'enquête ont été retirés. La population des enfants est donc un peu «biaisée ", au détriment des individus les plus qualifiés qui, pour certains, peuvent être encore en études à l'enquête. Cela explique sans doute le fait qu'il y ait légèrement plus d'ouvriers parmi les fils aînés que parmi les enquêtés. À partir de la génération enquêtée, les femmes deviennent proportionnellement plus nombreuses que les hommes parmi les professions intermédiaires et la féminisation de l'emploi s'accentue au fil des générations. On note également la forte baisse des ouvriers (proportion divisée par plus de deux entre la génération des pères et celle des enquêtés) et de l'inactivité féminine de génération en génération.

11. Cela s'explique sans doute par le fait que des professions qui regroupent beaucoup d'effectifs chez les femmes, comme les infirmières par exemple, sont classées différemment dans la nomenclature CNP (niveau A) et dans celle des PCS (professions intermédiaires).

12. Mais de nombreuses femmes de ces générations travaillaient aux côtés de leur époux indépendant, notamment dans les exploitations agricoles, sans être déclarées comme actives : voir, pour la France, Remillon et Lelièvre, 2018. 
TABLEAU 3

Répartition des PCS dans les lignées au Québec ${ }^{\mathrm{a} b \mathrm{c} d}$

\begin{tabular}{|l|c|c|c|c|c|c|c|}
\hline \multicolumn{1}{|c|}{ PCS } & Indépendant·e·s & Cadres & $\begin{array}{c}\text { Professions } \\
\text { intermédiaires }\end{array}$ & Employé.e.s & Ouvrier·e·s & Inactif/ve·s \\
\hline Position dans la lignée & \multicolumn{5}{|c|}{$\%$ pondérés } \\
\hline Père & 17,4 & 11,7 & 10,7 & 12,8 & 47,2 & 0,2 \\
\hline Mère & 3,3 & 1,6 & 7,9 & 14,7 & 7,8 & 64,7 \\
\hline Ego Homme & 1,3 & 27,5 & 21,9 & 25,0 & 21,5 & 2,8 \\
\hline Ego Femme & 0,4 & 18,1 & 24,7 & 44,5 & 3,7 & 8,5 \\
\hline Fils aîné & 1,7 & 27,5 & 23,5 & 22,5 & 22,6 & 2,2 \\
\hline Fille aînée & 1,9 & 19,3 & 37,0 & 33,4 & 0,8 & 7,7 \\
\hline
\end{tabular}

a Lignées des générations enquêtées (francophones, nés au Québec entre 1934 et 1954 et résidant en 2004 dans la région du Grand Montréal).

b L'interprétation de ce tableau se fait par exemple de la manière suivante: $11,7 \%$ des pères des Ego avaient une profession de cadre alors que ce n'était le cas que de 1,6\% des mères; $27,5 \%$ des Ego hommes sont dans cette situation, $18,1 \%$ des Ego femmes et, parmi les aînés des enfants, $27,5 \%$ des fils et $19,3 \%$ des filles. On voit donc une surreprésentation des hommes dans la catégorie «cadre», qui s’atténue au fil des générations.

c Nous avons exclu de la catégorie «inactif/ve·s» les aîné·e·s toujours en études.

d Tou·te·s les enseignant·e·s ont été regroupé·e·s avec les professions intermédiaires

Source: Enquête Biographies et solidarités familiales au Québec (Université de Montréal, 2004)

TABLEAU 4

Répartition des PCS dans les lignées en France ${ }^{\mathrm{b} b \mathrm{c}}$

\begin{tabular}{|l|c|c|c|c|c|c|c|}
\hline PCS & Indépendant·e·s & Cadres & $\begin{array}{r}\text { Professions } \\
\text { intermédiaires }\end{array}$ & Employé·e·s & Ouvrier·e·s & Inactif/ve·s \\
\hline Position dans la lignée & \multicolumn{7}{|c|}{$\%$ pondérés } \\
\hline Père & 26,5 & 15,0 & 11,4 & 13,6 & 32,2 & 1,4 \\
\hline Mère & 13,7 & 2,0 & 5,5 & 14,5 & 10,1 & 54,2 \\
\hline Enquêté & 9,0 & 29,6 & 20,6 & 9,3 & 31,5 & 0,0 \\
\hline Enquêtée & 4,7 & 17,1 & 21,8 & 44,1 & 7,8 & 4,6 \\
\hline Fils aîné & 5,6 & 26,0 & 26,7 & 15,8 & 22,7 & 3,1 \\
\hline Fille aînée & 2,7 & 21,0 & 33,4 & 33,2 & 2,0 & 7,8 \\
\hline
\end{tabular}

a Lignées des générations enquêtées (Individus nés entre 1930 et 1950 et résidant en Île de France en 1999).

b L'interprétation de ce tableau se fait par exemple de la manière suivante: en France, l'écart entre hommes et femmes était particulièrement fort pour les parents des générations enquêtées concernant la catégorie «cadres»: seules $2 \%$ des professions principales des mères étaient à ce niveau contre $15 \%$ des pères. L'écart entre les sexes se réduit, mais demeure, pour les générations suivantes: $29,6 \%$ des Ego hommes sont cadres à la fin de leur carrière contre $17,1 \%$ des Ego femmes, et c'est le cas, parmi les aînés des enfants, de respectivement $26 \%$ des fils et $21 \%$ des filles.

' Nous avons exclu de la catégorie «inactif/ve·s» les aîné·e·s toujours en études.

Source: Enquête Biographies et entourage (INED, 2001) 
Les constats sont globalement similaires côté français. On constate également une baisse de la proportion d'indépendant.e.s mais les indépendantes demeurent quand même plus nombreuses qu'au Québec (et l'étaient notamment plus dans la génération des mères). Comme précédemment, les femmes restent moins représentées que les hommes parmi les cadres, même si l'écart diminue de génération en génération. La proportion d'ouvriers était moins importante qu'au Québec dans la génération des pères, mais elle diminue aussi. La proportion d'inactives parmi les mères était également un peu inférieure en France par rapport au Québec, mais c'était la situation de la majorité des mères et la proportion d'inactives, en forte baisse dans les générations suivantes, atteint ensuite des niveaux comparables.

\subsection{Transmissions professionnelles dans les lignées}

Qu'en est-il à présent des transmissions professionnelles entre les femmes et les hommes des générations enquêtées et leurs parents, pères et mères, en France et au Québec? Dans le cas français, les mécanismes de reproduction sociale sont bien visibles notamment entre les hommes et femmes des générations enquêtées et leurs pères (figure 1). Ainsi, l'origine la plus représentée chez les enquêtés ${ }^{13}$ indépendants est père indépendant, père cadre chez les enquêtés cadres, père ouvrier chez les enquêtés ouvriers. Le constat est globalement le même entre les enquêtées et leurs pères. Les mères des générations enquêtées étaient majoritairement inactives ${ }^{14}$, encore plus pour les mères des enquêtés ouvriers ou employés. La distribution des professions des mères reflète davantage la structure de l'emploi des mères - beaucoup étaient inactives, sinon employées — que des mécanismes de reproduction sociale. On peut remarquer cependant que les hommes ouvriers ont plus fréquemment que les autres une mère qui était ouvrière et les cadres une mère cadre ou profession intermédiaire. Les indépendantes se distinguent également par le fait que leur mère était bien moins souvent inactive que celle des autres enquêtées et souvent indépendante ellemême, autant de signes de transmissions par les mères.

13. Par «enquêté·e » nous voulons désigner dans cette partie les hommes et les femmes de la génération enquêtée et non ceux/celles qui ont été effectivement échantillonné·e·s et interrogé·e·s. Tous les pourcentages des figures sont en effet pondérés et donc représentatifs de la population étudiée dans son ensemble (les Franciliens des générations 1930-1950).

14. Du moins leurs enfants - les personnes enquêtées — ont-ils déclaré que leur profession principale était « inactive ». Nous rappelons que, pour les besoins de la comparaison France-Québec, c'est cette " profession principale» que nous avons retenue ici car c'était la seule mesure de la profession disponible pour les parents dans l'enquête québécoise. Dans un autre article, centré sur la France, nous montrons cependant que ces mères ont très souvent travaillé à un moment donné de leur carrière et que retenir comme profession pour elles leur dernière profession d'active (plutôt que leur profession principale) conduit à mettre en évidence des mécanismes de transmissions professionnelles par les mères également (Remillon et Lelièvre, 2018). 
FIGURE 1

Transmissions dans les lignées en France Ego Hommes (\% pondérés) ${ }^{\mathrm{a}} \mathrm{b}$
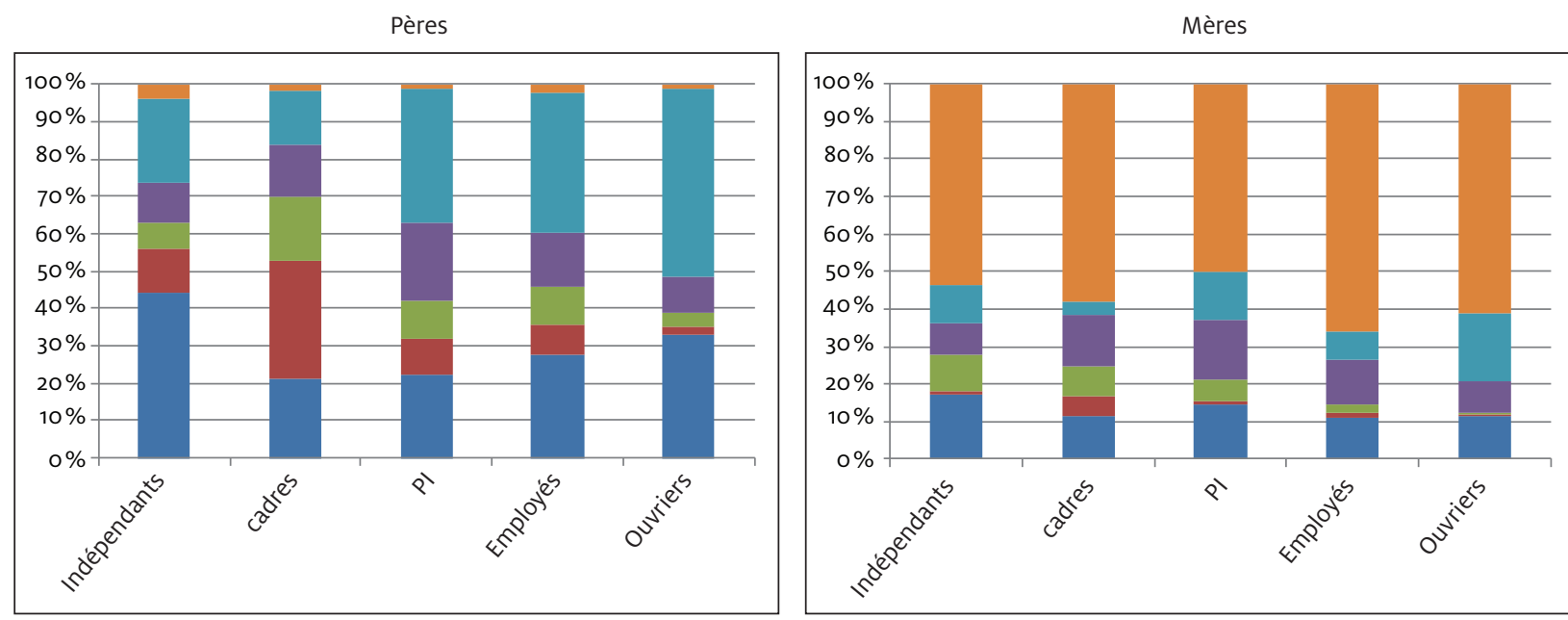

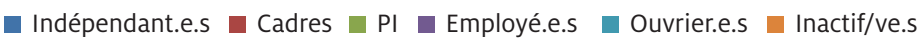

Transmissions dans les lignées en France Ego Femmes (\% pondérés)
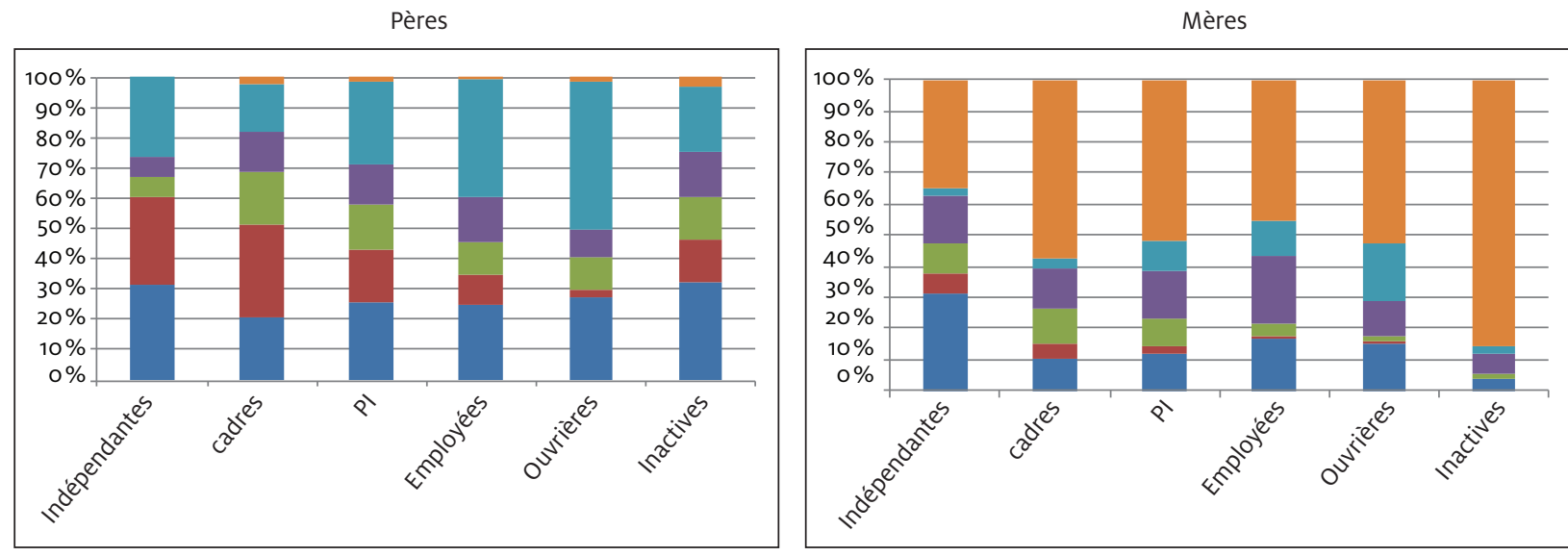

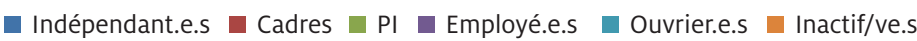

${ }^{a}$ Hommes et femmes des générations enquêtées (1930-1950 résidant en Île-de-France en 1999) et leurs parents.

b L'interprétation de cette figure se fait par exemple de la manière suivante: Parmi les Ego hommes de profession «indépendants», $44 \%$ ont déclaré que la profession principale de leur père était indépendant, 22,5\% ouvrier, 11,9\% cadre, etc. L'origine «père indépendant» est nettement surreprésentée chez les Ego hommes indépendants. C'est aussi l'origine la plus fréquente pour les Ego femmes indépendantes (31,5\%), suivie par un père cadre $(28,7 \%)$.

Source: Enquête Biographies et entourage (INED, 2001).

On perçoit également les mécanismes de reproduction sociale dans le cas québécois (figure $2^{15}$ ), mais ils apparaissent moins marqués que dans le cas français, en tous cas entre les enquêtés (hommes) et leurs pères. L'origine la plus fréquente chez tous les Ego hommes est en effet un père

15. Étant donné les faibles effectifs, certaines cases étaient trop petites (les indépendant·e·s dans la génération enquêtée, les inactifs chez les pères) et ont donc été enlevées. 
FIGURE 2

Transmissions dans les lignées Québec Ego Hommes (\% pondérés) ${ }^{\mathrm{a}} \mathrm{b}$
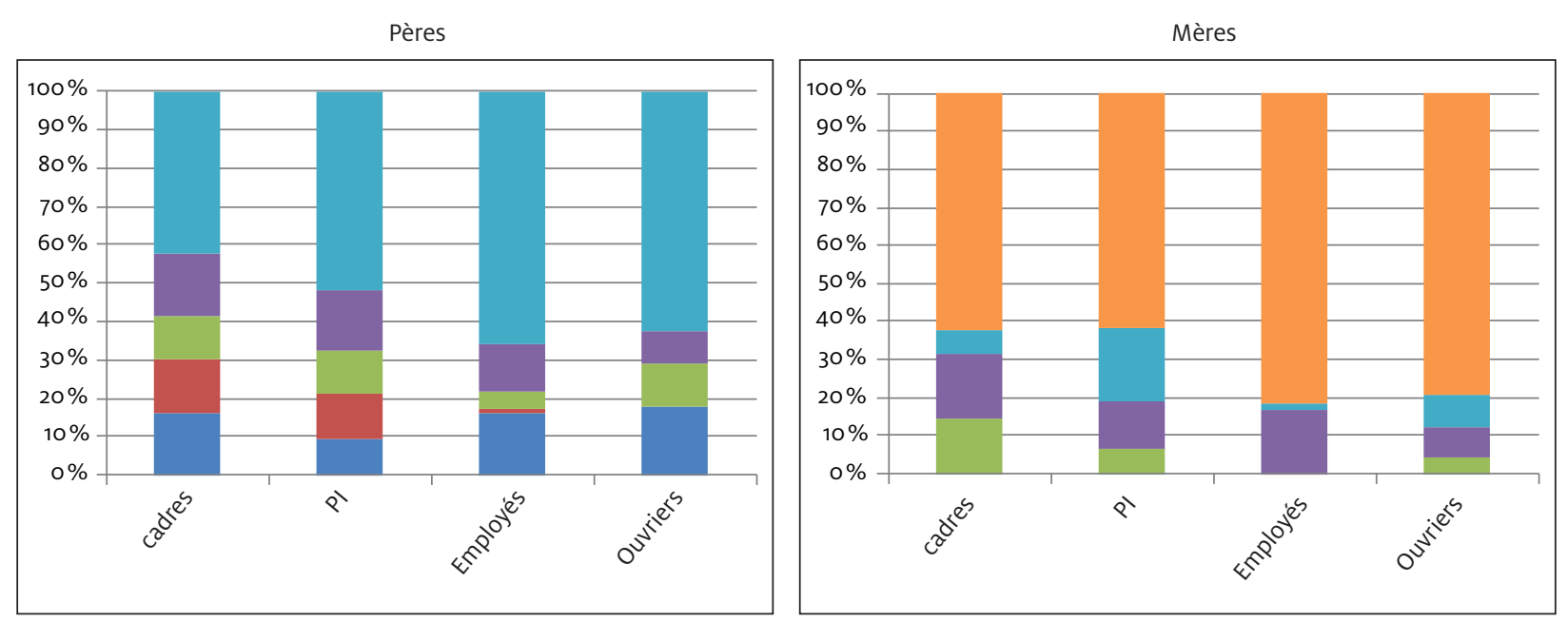

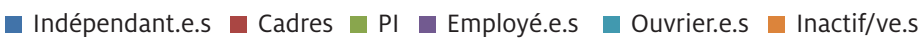

Transmissions dans les lignées Québec Ego Femmes (\% pondérés)
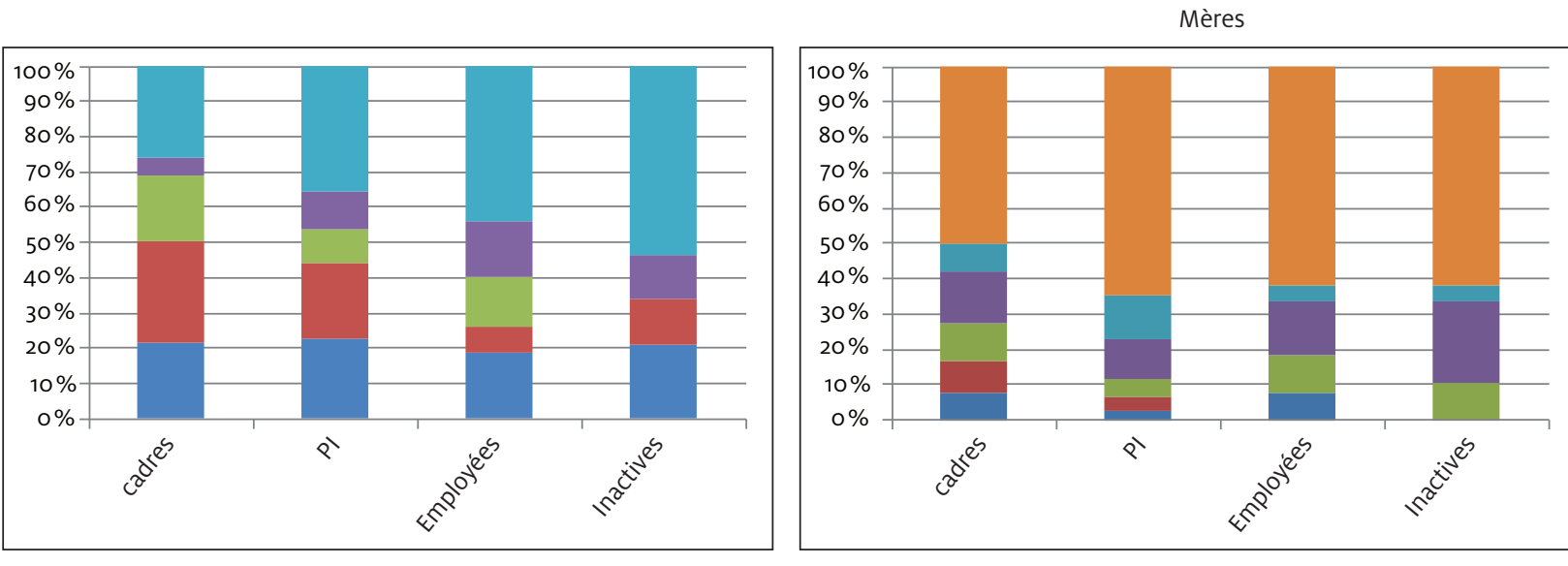

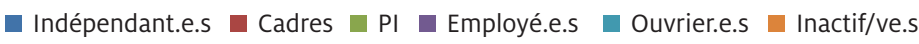

${ }^{a}$ Hommes et femmes des générations enquêtées (1934-1954, francophones nés au Québec résidant dans la région du Grand Montréal en 2004) et leurs parents.

b L'interprétation de cette figure se fait par exemple de la manière suivante: $29 \%$ des Ego femmes de profession cadre ont déclaré que leur père avait une profession principale de cadre, $26 \%$ ouvrier, $21 \%$ indépendant, etc. L'origine «père cadre» est surreprésentée chez les Ego femmes cadre. Ce n'est pas en revanche l'origine la plus fréquente pour les Ego hommes cadres (14\%). L'origine la plus fréquente pour eux est «père ouvrier » (42\%).

Source: Enquête Biographies et solidarités familiales au Québec (Université de Montréal, 2004).

ouvrier. On voit quand même que les hommes cadres et professions intermédiaires sont ceux qui ont le plus souvent un père cadre ou profession intermédiaire, et que les employés et ouvriers sont ceux qui ont le plus souvent un père ouvrier. 
Ces mécanismes de reproduction sociale apparaissent (paradoxalement) plus forts entre les enquêtées et leurs pères : notamment les femmes cadres ou professions intermédiaires sont une forte proportion à avoir un père cadre - $29 \%$ dans un cas, $21 \%$ dans l'autre — une origine proportionnellement plus fréquente pour elles que pour leurs homologues masculins (respectivement $14 \%$ et $12 \%$ ). Cela tend à montrer qu'au Québec, les femmes cadres ou professions intermédiaires ont plus fréquemment une origine paternelle similaire que les hommes québécois et les Françaises dans la même situation.

Comme dans le cas français, la majorité des mères québécoises étaient inactives. Or, les hommes cadres ou professions intermédiaires ont plus fréquemment que les autres une mère qui était active (souvent employée ou profession intermédiaire pour les cadres et employée ou ouvrière pour les enquêtés de profession intermédiaire). En outre, les seules à avoir des mères cadres dans leurs origines maternelles sont des femmes cadres ou professions intermédiaires, soulignant, là encore, les origines fortement sélectionnées de ces dernières.

\section{CONCLUSION}

L'objectif était de rendre compte des enjeux méthodologiques entourant l'étude de la mobilité sociale dans le contexte des mutations structurelles majeures de l'univers du travail en Occident, en particulier l'accession massive des femmes aux emplois rémunérés à partir des années 1960. Le cas de la France et du Québec a plus particulièrement retenu l'attention du fait de l'existence de données inédites recueillies de part et d'autre, ceci dans le cadre d'une recherche biographique sur l'évolution de l'entourage et des solidarités familiales durant le $\mathrm{XX}^{\mathrm{e}}$ siècle. Certes, pour véritablement comparer la France et le Québec en matière de fluidité sociale, il faudrait mettre en œuvre des modèles plus complexes que les statistiques descriptives présentées dans cet article, tenant compte des évolutions de la structure sociale. Cependant, à première vue, la reproduction sociale semble forte au Québec, tout comme en France. Le codage en "genres de compétences » a permis de mettre en évidence une ségrégation professionnelle importante entre les sexes au Québec. Le fait de mobiliser la nomenclature hiérarchique socioprofessionnelle française sur les données québécoises a également permis de montrer que les mécanismes de « plafond de verre » — masqués par la nomenclature des " niveaux de compétences» - étaient aussi importants au Québec qu'en France, même si les écarts entre femmes et hommes se réduisent de génération en génération. Une limite est que la nomenclature des PCS adoptée est particulièrement bien en phase avec les institutions françaises et moins avec la structure socioprofessionnelle canadienne. Alain Chenu (2012) évoque ainsi l'intraduisibilité de la catégorie française de «cadre » y compris au Québec. Le caractère heuristique d'un tel usage de la nomenclature française pour mener des travaux comparatifs entre la France et le Québec a néanmoins été mis en évidence. Toutefois, la nomenclature canadienne présente elle aussi un caractère heuristique, invitant à une classification moins biaisée selon le sexe que la nomenclature française.

Adopter une nomenclature socioprofessionnelle commune pour comparer les deux enquêtes s'est avéré riche d'enseignements. Nous avons montré que, pour la génération des hommes enquêtés (couvrant en partie les baby-boomers), la reproduction sociale passait surtout par les pères - encore plus fortement en France qu'au Québec car, au Québec, l'origine la plus fréquente 
est « père ouvrier » pour toutes les catégories socioprofessionnelles. Les transmissions des pères à la génération des femmes enquêtées sont fortes également, notamment pour les Québécoises cadres et professions intermédiaires qui ont très fréquemment un père cadre. Les transmissions venant des mères sont moins visibles — car, des deux côtés de l'Atlantique, la majorité ont été déclarées « inactives " par les personnes enquêtées — mais non nulles, avec notamment des mères plus fréquemment actives chez les personnes enquêtées aux positions socioprofessionnelles élevées, en France et encore davantage au Québec. Pour creuser ces transmissions par les mères, un prolongement de ce travail sera d'analyser ce qu'il en est de ces transmissions intergénérationnelles non plus entre deux mais entre trois générations (parents, Ego et enfants) comme nous l'avons fait pour le cas français (Remillon et Lelièvre, 2018).

\section{BIBLIOGRAPHIE}

AMOSSÉ, T. 2004. "Professions au féminin. Représentation statistique, construction sociale ", Travail, Genre et Sociétés, 11 : 31- 46.

AMOSSÉ, T. 2012. "Catégories socio-professionnelles : quand la réalité résiste! Après le crépuscule, une aube nouvelle? », Revue française de Socio-Économie, 2 : 225-234.

AMOSSÉ, T. 2013. "La nomenclature socio-professionnelle une histoire revisitée ", Annales. Histoire, Sciences Sociales, 4:1039-1075.

BEAUD, J.P. et J. G. PRÉVOST. 1992. «La classification canadienne des occupations pendant l'entre-deux-guerres : réflexion sur un cas d'indépendance statistique », Canadian Journal of Political Science/Revue canadienne de science politique, 25, 3: 489-512.

BOLTANSKI, L. et L. THÉVENOT. 1983. "Finding one's way in social space : a study based on games », Social Science Information, 22, 4/5:631-680.

BONVALET, C. et E. LELIEVRE. 2012. De la famille à l'entourage. Paris, Collection Les Grandes Enquêtes, Editions de l'INED/ PUF, 472 p.

BROUSSE, C. 2012. "Réflexions sur la nomenclature socio-économique européenne en gestation ", Revue française de socio-économie, 2, $10: 241-250$.

CHAUVEL, L., A. CHENU, M. OBERTI et E. PRÉTECEILLE. 2002. «Enjeux et usages des catégories socioprofessionnelles : traditions nationales, comparaisons internationales et standardisation européenne ", Sociétés contemporaines, 1, 45-46:157-185.

CHENU, A. 2012. "Les catégories socioprofessionnelles : sous la nomenclature, quelles institutions? », Revue Française de Socio-Economie, 2, 10 : 235-240.

CHEVRIER, C.et D-G. TREMBLAY. 2003. "Portrait actuel du marché du travail au Canada et au Québec : une analyse statistique en fonction du genre ", Note de recherche de la Chaire de recherche du Canada sur les enjeux socio-organisationnels de l'économie du savoir, 2003-02, 52 p.

CORAK, M.et A. HEISZ. 1999. "The Intergenerational Earnings and Income Mobility of Canadian Men : Evidence from Longitudinal Income Tax Data », The Journal of Human Resources, $34,3: 504-533$.

FILHON, A., J. DEAUVIEAU, L. DE VERDALLE, A. PELAGE, T. POULLAOUEC, C. BROUSSE, M. MESPOULET et K. SZTANDAR-SZTANDERSKA. 2013. "Un projet de nomenclature 
socioprofessionnelle européenne : une construction savante face aux variations nationales de représentation du monde social », Sociologie, 4:373-393.

GAUTHIER, H. 2001. "Chapitre 10. Les professions », dans Portrait social du Québec, données et analyses, édition $2001: 245-265$.

KEMPENEERS, M., E. LELIEVRE et N. ROBETTE. 2015. «Trajectoires d'activité des femmes issues du baby-boom : une comparaison France-Québec», dans C. BONVALET et collab. (Eds.), Les baby-boomers, une histoire de familles, Montréal, Presses Universitaires du Québec : 127-144.

KEMPENEERS, M. et I. VAN PEVENAGE. 2011. " Les espaces de la solidarité familiale », Recherches sociographiques, 52, $1: 105-119$.

KEMPENEERS, M. et I. VAN PEVENAGE. 2013. "Biographies and family solidarity in Québec ", International Review of Sociology, 23, 1:85-103.

LANGLOIS, S. 2003. "Quatre décennies d'études sur la stratification sociale au Québec et au Canada : tendances et illustrations ", Lien social et politiques, 49: 45-70.

LAROCHE, D. 1997. "La mobilité sociale au Québec », Statistiques sociales. D’une génération à l'autre, évolution des conditions de vie, vol. 2 : 165-204.

MARCHAND, O. 1993. "Les emplois féminins restent très concentrés », Données sociales, Paris, Insee.

MARUANI, M. 2003. Travail et emploi des femmes. Paris, La Découverte, 128 p.

MERON, M., M. AMAR, C. BABET, M. BOUCHET-VALAT, F. BUGEJA-BLOCH, F. GLEIZES, F. LEBARON, C. HUGRÉE, E. et A. SPIRE. 2016. "ESeG=European Socio economic Groups", Série des Documents de Travail de la Direction des Statistiques Démographiques et Sociales, $\mathrm{n}^{\circ} \mathrm{F} 1604$.

MOULIN, S. 2016. Inégalités : mode d'emploi. L'injustice au travail au Canada. Québec, Canada, Les Presses de l'Université de Montréal, 349 p.

MOULIN, S., A. DUPRAY et R. D’AMOUR. 2013. "Les politiques publiques au cour des bifurcations individuelles ", Sociologie et Sociétés, 45, 1: 203-228.

PENISSAT, E. 2012. "La difficile production d'une nomenclature socioprofessionnelle à l'échelle européenne », Revue française de socio-économie, 2, 10 : 251-257.

PENISSAT, E., C. BROUSSE et J. DEAUVIEAU. 2015. " Finding one's way in social space : genèse, postérité et actualité d'une enquête originale », Sociologie, 1: 31-42.

PIERRU, E. et A. SPIRE. 2008. "Le crépuscule des catégories socioprofessionnelles ", Revue française de science politique, 58, $3: 457-481$.

REMILLON, D. et E. LELIÈVRE. 2018. "Positions et transmissions socioprofessionnelles des femmes et des hommes au sein de lignées franciliennes », Travail et emploi, $\mathrm{n}^{\circ}{ }_{154}$, avril-juin : 43-70. 


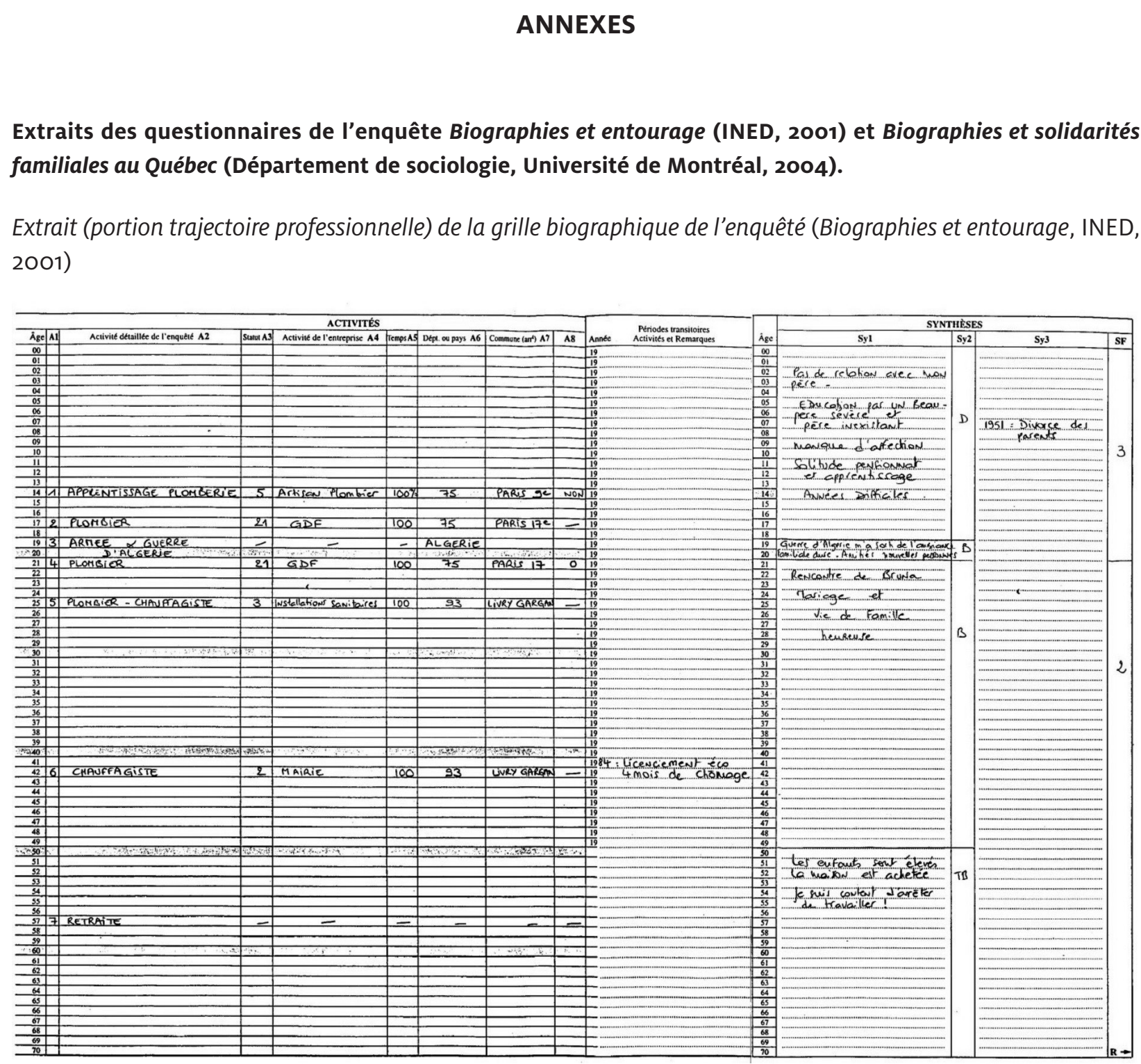

Note: L'enquêté retraité à 57 ans, a débuté son apprentissage de plomberie à 14 ans. II devient plombier à 17 ans, pour Gaz de France (GDF), puis doit s'interrompre à 18 ans pour faire l'armée et la guerre d'Algérie. À son retour, à 21 ans, il retrouve son emploi de plombier chez GDF, qu'il quitte pour un emploi salarié de plombier-chauffagiste à 25 ans à Livry-Gargan où il réside. Après son licenciement économique et 4 mois de chômage, en 1984, il retrouve à la mairie de Livry-Gargan un emploi de chauffagiste qu'il occupera jusqu'à sa retraite. 
Grille biographique de la mère de l'enquêté (Biographies et entourage (INED, 2001))

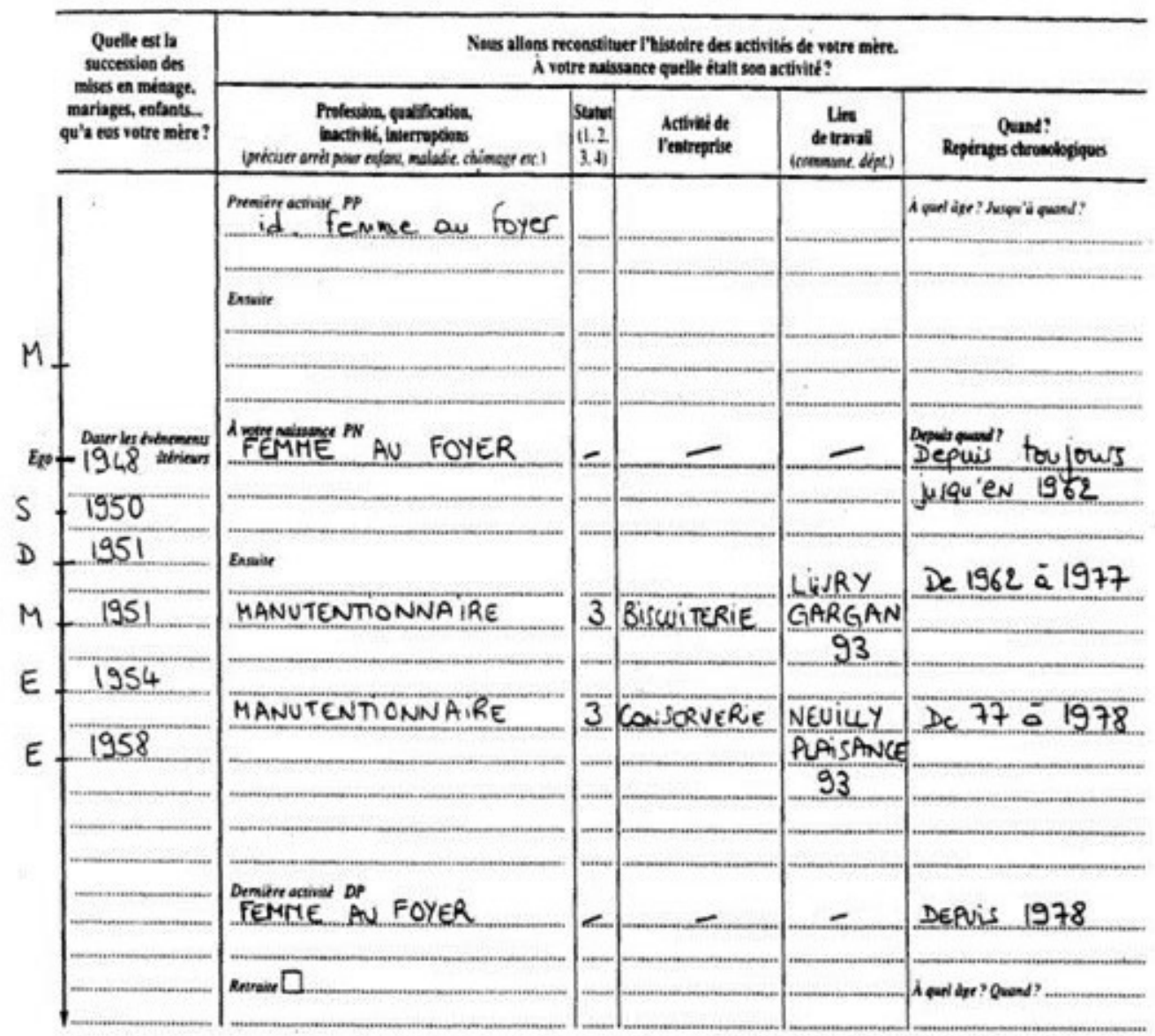

- A-t-elle connu des périodes d'inactivitét ou des interruptions dues da la guerre, au chômage, à la maladie, aux enfants, à une reprise d'études...? Si ouli, les placer dans la chronologic.

\section{Récapitulons : pour voes quelle a eté son activité principale (préciser la qualification, $O S, O Q$..)?} FEMME AU FOYER

1. Independante

3. Salanite secieur prive

2. Salanite du secteur public et nationalist (priciser).

4. Autre (préciser)

Activite de son entreprise :

(ex: parfemerie: fabricarion ou commenee) 
Extrait (portion trajectoire professionnelle) de la grille biographique de l'enquêté (Biographies et solidarités familiales au Québec (Département de sociologie, Université de Montréal, 2004))

\begin{tabular}{|c|c|c|c|c|c|c|c|c|}
\hline Année & Age & $\begin{array}{r}\text { Etudes, em } \\
\text { d'interrup } \\
\text { pour e }\end{array}$ & $\begin{array}{l}\text { aplois occupés } \\
\text { ptions et d'ina } \\
\text { enfant, maladie }\end{array}$ & $\begin{array}{l}\text { is (profession), périodes } \\
\text { activités (Préciser arrêt } \\
\text { ie, chômage, etc...) }\end{array}$ & $\begin{array}{c}\text { à } \\
\text { son compte / } \\
\text { Salariế }\end{array}$ & $\begin{array}{l}\text { Type de contrat } \\
\text { Temps pleins } / \\
\text { Temps partiel }\end{array}$ & $\begin{array}{c}\mathrm{Nb} \\
\text { employés }\end{array}$ & Nom, activité et lieu de l'entreprise \\
\hline 19449 & 00 & . & & 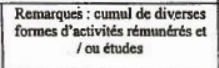 & & & & \\
\hline 1950 & 01 & & & & & & & \\
\hline 1951 & 02 & & & & & & & \\
\hline 1952 & 03 & & & & & & & \\
\hline 1953 & 04 & & & & & & & \\
\hline 1954 & 05 & & & & & & & \\
\hline 1955 & 06 & & & & & & & \\
\hline 1956 & 07 & ttudesy & & & & & & \\
\hline 1957 & 08 & & & & & & & $=$ \\
\hline 1958 & 09 & & & & & & & \\
\hline 1959 & 10 & & & & 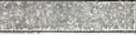 & 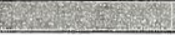 & 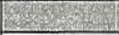 & 20 \\
\hline 1960 & 11. & & & & & & & \\
\hline 1961 & 12 & & & & & & & \\
\hline 1962 & 13 & & & & & & & \\
\hline 1963 & 14 & & & & & & & \\
\hline 1964 & 15 & & & & & & & \\
\hline 1965 & 16 & $\downarrow$ & & & & & & \\
\hline 1966 & 17 & ETUDES & & & & & & \\
\hline 1967 & 18 & TECHNicie & EN to $A R$ & RPENTACE & peiture & peleir & Nsp & TENDRON-TEFE URE - LAVAL-QQC \\
\hline 1968 & 19 & & & & & & & \\
\hline 1969 & 20 & & 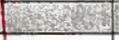 & 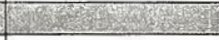 & 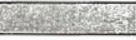 & 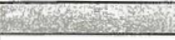 & Fenter & 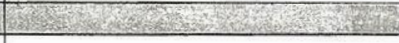 \\
\hline 19.70 & 21 & T1 & & & & & & \\
\hline 1971 & 22 & $\begin{array}{ll}y & 1\end{array}$ & 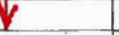 & & & & & \\
\hline 19 极 & 23 & 4 & & $"$ & " & II & 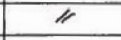 & HYDRO-QUEBEC - MONTREAC \\
\hline 1913 & 24 & WNSTRNCTER & OR DE UA & 1 & salasié & plain & Nsp & SET THERESE AVIATION- STE \\
\hline 1974 & 25 & PROTE D & 28 B Bousse & 1 & Aalarié & Desin & Nop. & ARR BRAZEAU - ROUYN NORAADAA \\
\hline 19.75 & 26 & & & & & & & \\
\hline 1976 & 27 & & & & & & & \\
\hline 19.77 & 28 & $y$ & & & & & & \\
\hline 19.78 & 29 & PILOTE $D E$ & ELIGNE & 1 & Aolatie & peein & $\simeq 22000$ & ARR CANADA - MONTREALERAL \\
\hline 19,9 & 30 & 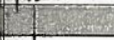 & 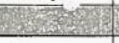 & 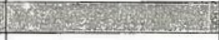 & 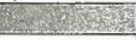 & 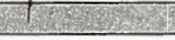 & 20 & 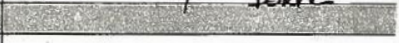 \\
\hline 1990 & 31 & & & & & & & \\
\hline
\end{tabular}

Note: L'enquêté a arrêté ses études et commencé à travailler à 18 ans comme technicien en arpentage, dans deux entreprises, d'abord à Laval puis à Montréal. II bifurque progressivement vers le secteur aéronautique et devient pilote de ligne pour Air Canada à 29 ans. 
Grille biographique du père de l'enquêté (Biographies et solidarités au Québec (Département de sociologie, Université de Montréal, 2004))

Quelle est la succession des événements familiaux : mise en ménage, mariage, enfants, etc., qui jalonnent la vie d'adulte de votre père?

(les noter en ordre chronologique)

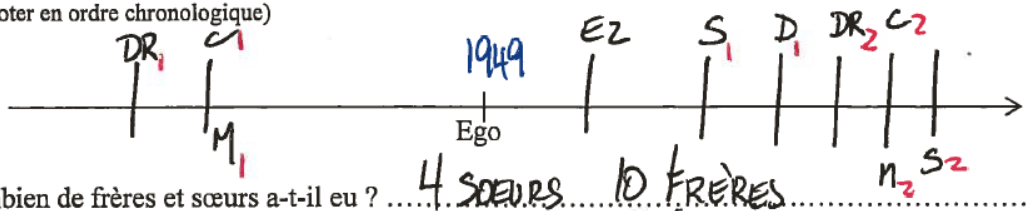

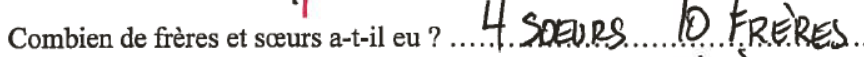

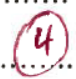

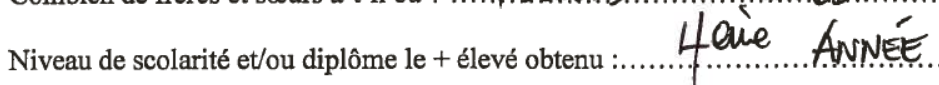

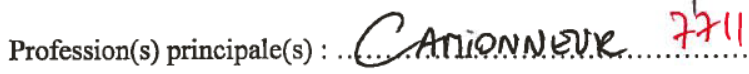

A son compte ou salarié ?....... SAIARTE

Fréquence de vos contacts avec lui (actuellement décès)

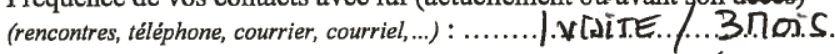

...Apper. .... Senniné. 